\title{
Single-fraction low-energy electronic brachytherapy for conjunctival lymphoma
}

\author{
Gustavo R. Sarria, MD!, Carla M. Cabrera, MD², Prof. Gustavo J. Sarria, MD², Mario Buitrago, MD³, Paola Fuentes, MD², \\ Solon Serpa, MD³, Frank A. Giordano, MDl \\ 'Department of Radiation Oncology, University Medical Center Mannheim, Medical Faculty Mannheim, University of Heidelberg, Mannheim, \\ Germany, ${ }^{2}$ Radiotherapy Department, Instituto Nacional de Enfermedades Neoplasicas, Lima, Peru, ${ }^{3}$ Ophthalmic Oncology Department, \\ Instituto Nacional de Enfermedades Neoplasicas, Lima, Peru
}

\begin{abstract}
Purpose: Conjunctival lymphoma represents an uncommon tumor, accounting for $5-10 \%$ of total extranodal lymphomas. Although radiotherapy is a frequent treatment option, limited capacities and lack of specialized centers are common problems in Peru, forcing radiation oncologists to apply short courses of radiotherapy. Here, we report a case series of patients treated with a novel single-shot scheme. Additionally, we present a literature review of the current short-course irradiation strategies.

Cases presentation: Three cases of conjunctival marginal zone (B-cell) lymphoma (marginal zone lymphoma [MZL]/mucosa-associated lymphoid tissue [MALT]) of the fornix are presented. Following biopsy and sonographic assessment of the lesion thickness, we applied a focused single dose of 14 Gy kilovoltage brachytherapy (prescribed to the maximum thickness of the lesion). Follow-up was scheduled in quarterly intervals. After 28,31 , and 40 months of follow-up, none of the three patients treated exhibited acute or chronic toxicities and remained local or distant disease-free.

Conclusions: Single dose kilovoltage brachytherapy was effective and safe in this small cohort of patients. Based on the literature, there is an evidence that local treatment in short-course radiotherapy is effective and should be considered amongst therapeutic options for these patients; however, this novel approach should be evaluated prospectively in a larger cohort.

J Contemp Brachytherapy 2020; 12, 3: 267-272 DOI: https://doi.org/10.5114/jcb.2020.96869
\end{abstract}

Key words: electronic brachytherapy, ocular adnexa lymphoma, short-course radiotherapy.

\section{Purpose}

Lymphomas of the ocular adnexa are infrequent entities, with roughly $8 \%$ of extranodal lymphomas [1]. The most affected subsites are the conjunctiva, lacrimal glands, and eyelids. Different reports show that the most common histological type is of B-cell origin (98\%), of these, the most frequent subtype is extranodal marginal zone lymphoma (MZL; 80\%), also known as mucosa-associated lymphoid tissue (MALT) lymphoma, followed by follicular lymphoma ( $8 \%$ ), diffuse large B-cells lymphoma $(3 \%)$, and mantle cell lymphoma (3\%) [2,3,4]. Conjunctival lymphoma is a neoplasm predominantly affecting older adults. Low-grade subtypes such as MZL usually appear around the age of 60 , while high-grade subtypes are more frequent in patients older than 70 years $[5,6,7]$.

To date, no standard of care have been established, as only few heterogeneous data are available on how to treat these tumors [8]. As local recurrence is the most common pattern of failure, local radiotherapy is a frequently ap- plied modality, resulting in local control rates of $33-100 \%$ after 5 years, with a wide variable range due to patient selection and different treatment modalities [9]. However, details on radiation techniques, radiation doses, and field definitions required to achieve local control remain unclear.

Radiation-sensitive structures such as retina and cornea, limit the doses and field sizes in conventional external-beam radiotherapy (EBRT). In turn, surface brachytherapy using low-energy X-rays may allow sparing organs at risk, as they are rapidly absorbed with a steep dose fall-off and thus, only few millimeters of tissue receives therapeutic doses and organs-at-risk can be spared [10].

Here, we present the outcomes of three patients diagnosed with ocular conjunctival lymphoma treated with low-energy brachytherapy. Due to a unique physical characteristics of this X-rays, efficient sparing of healthy tissues allowed to apply a single therapeutic fractions.
Address for correspondence: Gustavo R. Sarria, MD, Department of Radiation Oncology, University Medical Center Mannheim, Medical Faculty Mannheim, University of Heidelberg, Theodor-Kutzer-Ufer 1-3, 68167 Mannheim, Germany, phone: +49-621-383-6020, fax: +49-621-383-3493, e-mail: gustavo.sarria-vargas@umm.de
Received: 08.10.2019

Accepted: 12.12 .2019

Published: 30.06 .2020 
We also reviewed and discussed other studies and case series where single-fraction or short-course regimes were used.

\section{Cases presentation}

Case no. 1 is a 67-year-old woman that presented with foreign body sensation, redness, and pain in the left eye. A physical exam showed a $20 \times 10 \mathrm{~mm}$ conjunctivaattached highly vascularized lesion of salmon color, reaching from the bulbar conjunctiva to the lower fornix. The visual acuity was 20/25 for the right eye and 20/40 for the left eye; ocular motility was preserved, pupils were reactive to light, and a fundus exam showed no abnormal findings. An ocular ultrasound revealed a lesion thickness of $2 \mathrm{~mm}$. The final pathology as per incisional biopsy revealed the diagnosis of extranodal marginal zone lymphoma (MZL/MALT) of a CD20+, CD5-, BCL6-, and CD3- phenotype, with low proliferative activity (Ki67 of 10\%) and a restriction to light chains (KAPPA+/ LAMBDA-). Further staging with chest and abdominal tomography and bone marrow aspiration was negative, which was consistent with a clinical stage IE, and local brachytherapy was estimated to be sufficient to control this disease. The application procedure was then carried out under topical anesthesia, blocking of the ocular musculature, and fixing of the eyelids with a blepharostat. The

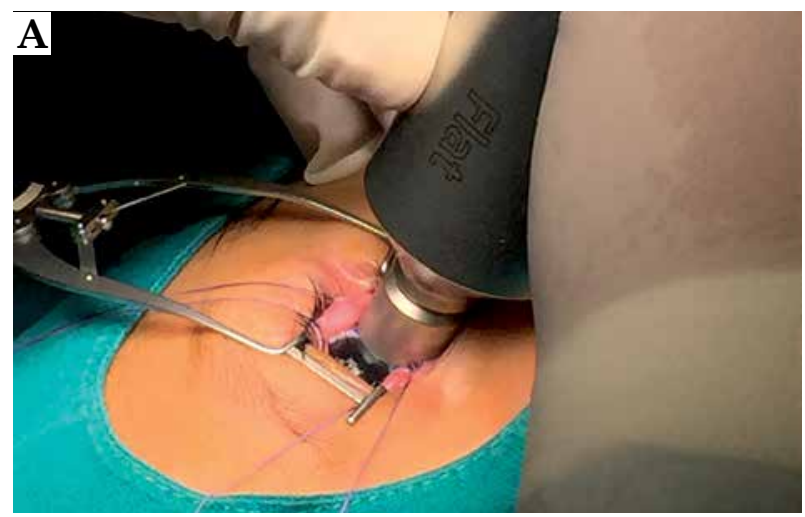

Fig. 1. Application procedure

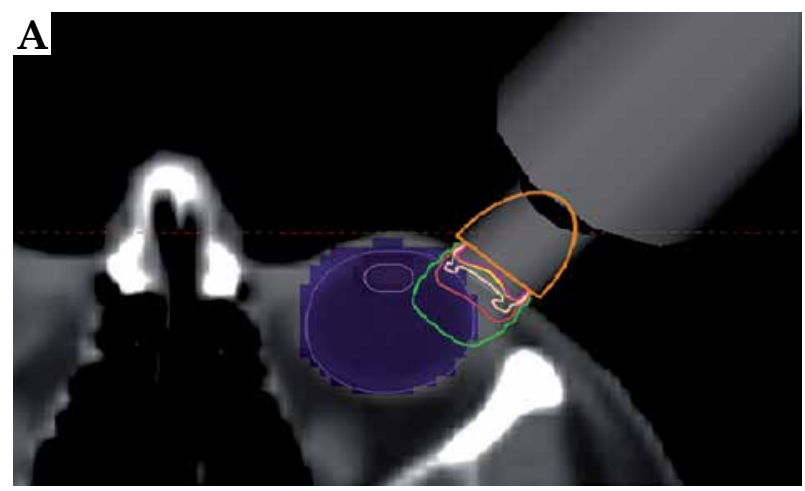

Fig. 2. Maximum dose to lens after $3 \mathrm{~mm}$ depth prescription head was then fixed in a thermoplastic mask, which was fenestrated over the left eye. For brachytherapy, a low-energy X-ray device (50 kV maximum energy; Intrabeam, Carl Zeiss Meditec AG, Jena, Germany) equipped with a $2 \mathrm{~cm}$ flat applicator was placed on the lesion, with light pressure applied to flatten the tumor. Irradiation with 14 Gy prescribed to a $2 \mathrm{~mm}$ depth was started once the articulable arm of the device was fixed (Figure 1). The dose was chosen after calculating the equivalent dose of a $2 \mathrm{~Gy}$ fraction scheme $\left(\mathrm{EQD}_{2}=\mathrm{BED} /[1+\{2 / \alpha / \beta\}]\right)$, considering an approximate relative biological effectiveness (RBE) of 1.3 [10] and a tumor $\alpha / \beta$ ratio of $10 \mathrm{~Gy}$ [11], resulted in an $\mathrm{EQD}_{2}$ of $36.4 \mathrm{~Gy}$. Due to the absorption properties of low-energy X-rays, the dose to the lens was less than 4 Gy in the worst-case scenario (Figure 2). Thereafter, the patient presented in three-monthly follow-ups, where the visual acuity of treated eye was seen to be unaltered, and no adverse effects related to the local treatment were noted. Till now, no evidence of persistent or local recurrence after 3 years and 4 months are noted (Figure 3 ).

Case no. 2 is a 50 -year-old woman, with a 4 -month history of left eye redness and mild foreign body sensation. At the clinical examination, a salmon-colored conjunctival lesion was observed in the left eye at the level of lower fornix, with soft consistency, and encompassing the entire fornix. Visual acuity was unaltered, pupils were reactive, and ocular motility was preserved. An
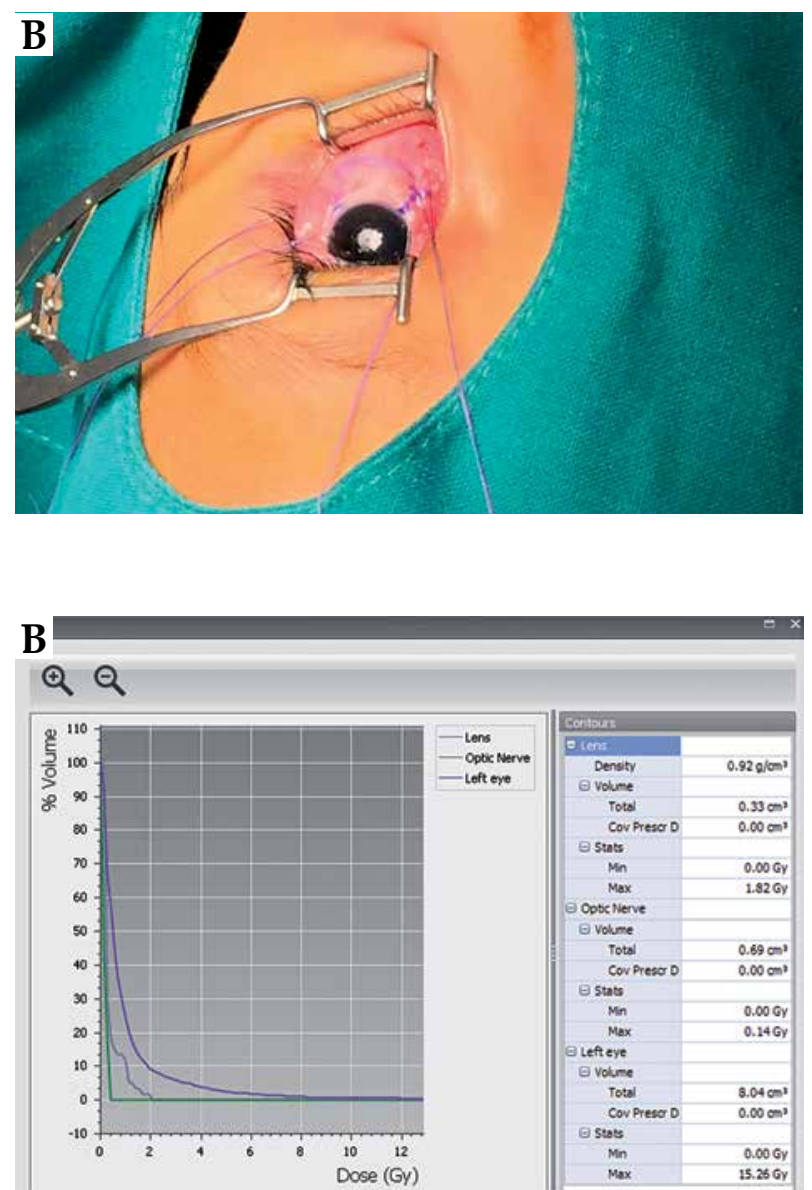

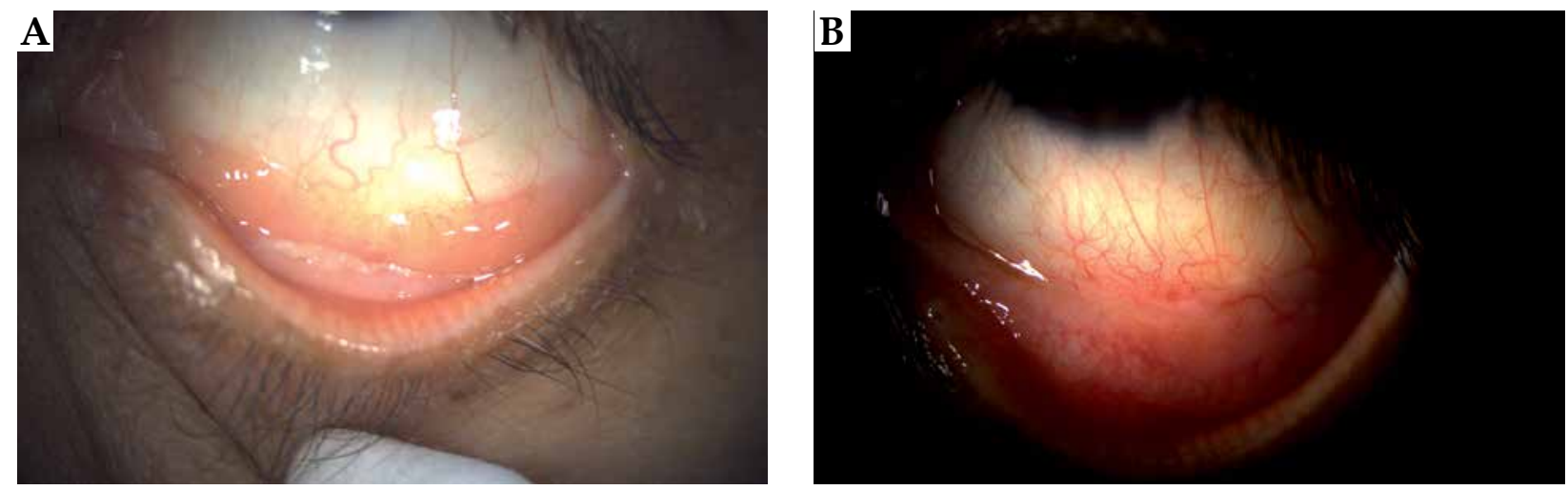

Fig. 3. Pre- and 2-year post-treatment control pictures show complete resolution of the lesion

ocular ultrasound showed a lesion thickness of $3 \mathrm{~mm}$. An incisional biopsy of the conjunctival lesion revealed a low-grade non-Hodgkin MALT lymphoma of a CD20+, BCL6-, CD5-, and CD3- phenotype, with a proliferative activity (Ki67) of 15\%. After further investigation, the patients were staged as IE. Superficial brachytherapy was performed with low energy photons at a dose of $14 \mathrm{~Gy}$, prescribed at $3 \mathrm{~mm}$ depth with a $2 \mathrm{~cm}$ flat applicator, as described for case No. 1. Quarterly controls were scheduled thereafter and, at a follow-up time of 2 years and 4 months, no evidence of disease recurrence or toxicity in the affected eye were noted.

Case no. 3 is a 67-year-old woman, who presented with a foreign body sensation in the left eye. On clinical examination, a conjunctival lesion was noted at the lower fornix, salmon-colored, with soft consistency. Visual acuity was unaffected on both eyes, pupils were reactive, ocular motility was preserved, and a fundus exam showed no abnormalities. The tumor thickness was $2 \mathrm{~mm}$ as per ocular ultrasound. An incisional was performed, establishing the diagnosis of extranodal marginal zone lymphoma of a CD20+, CD10-, BCL2+, CD5-, and CD3phenotype. Further staging $(\mathrm{CT}$, bone marrow aspiration) was negative, which was consistent with stage IE. Superficial brachytherapy was applied at a dose of 14 Gy, prescribed to $2 \mathrm{~mm}$ depth using a $1 \mathrm{~cm}$ flat applicator.
Three-monthly exams were then scheduled, revealing no evidence for recurrence or toxicity at a follow-up time of 2 years and 7 months.

\section{Discussion and conclusion}

Conjunctival lymphomas constitute the most frequent malignant tumors of the orbit, with the majority involving the conjunctiva with $50 \%$ of lesions located in the fornix and, to a much lower percentage, in the bulbar area, and rarely in the limbal or tarsal zone $[12,13,14]$. Their incidence is increasing and, due to regional differences, risk factors such as chronic infection with Chlamydia psittaci and Helicobacter pylori were discussed [4].

Patients with conjunctival lymphomas most frequently present with foreign body sensation and redness of the affected eye [5]. Other associated symptoms, although less common, are dry eye, epiphora, secretions, ectropion, or photophobia. B symptoms (fever, diaphoresis, weight loss) are rare in conjunctival lymphoma and could indicate a different phenotype $[15,16]$. The usual time span between first symptoms and first ophthalmological consultation is 1 month, although there are studies reporting up to 10 years of evolution prior to diagnosis [12].

MALT extranodal marginal zone lymphomas are not exclusively unilateral; there is a $4: 1$ relation to bilateral

Table 1. Reports on short-course radiotherapy for conjunctive lymphoma. The outcomes after 5 or less fractions of radiotherapy to treat conjunctival lymphoma are presented

\begin{tabular}{lcccc} 
Report/reference & $\begin{array}{c}\text { No. of } \\
\text { patients }\end{array}$ & $\begin{array}{c}\text { Total } \\
\text { dose }\end{array}$ & $\begin{array}{c}\text { Number of } \\
\text { fractions }\end{array}$ & Outcome(s) \\
\hline Sarria et al., 2018 & 39 & $18-22$ Gy & 1 & No occurrence of $\geq$ grade 2 toxicity \\
\hline Marr et al., 2015 & 2 & 15 Gy & 1 & $100 \%$ of local control at 24.9 months \\
\hline Regueiro et al., 2002 & 10 & $40-80$ Gy & 1 (LDR) & $67 \%$ of local control at 120 months \\
\hline This report & 3 & 14 Gy & 1 & $100 \%$ of local control at 26 months \\
\hline Bianciotto et al., 2010 & 13 & $13.5-22.5$ Gy & 5 & $100 \%$ of local control at 23 months \\
\hline Cotting-Bodmer et al., 2016 & 1 & 4 Gy & 2 & Local failure after 9 months \\
\hline Fasola et al., 2013 & 20 & 4 Gy & 2 & 85\% of local control at 26 months \\
\hline Hoskin et al., 2014 & 614 & 24 Gy vs. 4 Gy & 2 & 4 Gy was inferior to 24 Gy
\end{tabular}


presentation [17]. Although less frequent, systemic spread may occur and staging should therefore not be different from that of other lymphomas, with whole-body imaging studies as well as bone marrow exams [18]. In consequence, dependent on histology, the extent of the disease, prognostic factors related to the patient, and visual acuity of the affected eye, therapeutic options vary from localized to systemic therapy.

Localized conjunctival lymphoma may be either treated with EBRT, brachytherapy (BT), local immunotherapy, cryotherapy, or surgery [13]. Even observation has been suggested in selected patients [14]. Within the spectrum of radiotherapy tools, EBRT and BT are the most widespread options, with numerous published studies reporting control rates between $86 \%$ and $100 \%$ [19].

Conventional management with EBRT consists of delivering doses between 25 and 30 Gy in conventional fractionation (1.8-2 Gy per fraction) for indolent lymphomas, or up to $36 \mathrm{~Gy}$ for aggressive phenotypes. Electron beams, covering the entire conjunctiva and eyelids, are to date the most commonly used [9]. However, due to their specific absorption properties, complication rates with electrons are high, with a considerable fraction of patients developing xerophthalmia, retinal damage, cataract, and corneal ulcers $[20,21,22]$.

A different approach was described by Bianciotto et al. using short-course robotic fractionated-radiosurgery (CyberKnife). with doses between 13.5 and $22.5 \mathrm{~Gy}$ in 3 to 5 fractions. A complete response was noted in all of the 13 patients reported, after a mean follow-up of 23 months, with only one patient developing cataract after the treatment [23].

Brachytherapy is another commonly applied therapy in the management of this group of patients. There is a body of literature on favorable results with the use of isotopes either as plates, such as strontium, phosphorus, and iodine $[24,25,26]$, or isotopes coupled to an (anti-CD20) antibody [27]. A favorable experience shared by Regueiro et al. has shown local control rates of $76.9 \%$ ( $n=10 / 13$ eyes) without an evidence of failure in $67.5 \%$ of patients, who underwent treatment with ${ }^{90} \mathrm{Sr}$ and ${ }^{90} \mathrm{Y}$, to total doses between 40 and $80 \mathrm{~Gy}$, and after a median follow-up of 78 months. Despite these encouraging outcomes, $95 \%$ of their cohort developed G2 or higher toxicity, having 6 G3 or G4 events [26]. Although, not comparable regarding the number of patients presented here, our approach might potentially resemble fewer toxicity events than that aforementioned. From a logistics perspective, the use of unsealed source radiotherapy for conjunctival malignancies has declined in recent years, given the emergence of safer therapeutic alternatives for both, patients and health personnel.

Short-course and low-doses schemes have been described as well. A Stanford-based group published a series of 27 patients with stage I/II treated with only 4 Gy delivered in 2 fractions. This low-dose therapy resulted in 23 complete responses, 3 partial responses, and 1 stable disease after a median follow-up of 26 months [28]. A further single-patient case report also described this treatment scheme; however, after 9 months follow-up, the patient relapsed and received salvage re-irradiation of 25.2 Gy at 1.8 Gy per fraction [29].

Similar experience was described by König et al. in two consecutive papers. In the first retrospective review paper, they showed a group of 52 patients diagnosed with indolent orbital lymphomas between 1987 and 2014, comparing a total of 52 lesions treated with conventional radiotherapy schemes (26-46 Gy at 2 Gy per fraction) with a total of 8 lesions treated with a short-course radiotherapy scheme (4 Gy in 2 fractions). After a median follow-up of 133 months in the conventional radiotherapy group and 25 months in the short-course radiotherapy group, they described a 2-year progression-free survival (PFS) of $95 \%$ vs. $68.6 \%$, respectively [30]. In a subsequent paper, they presented the outcomes of 47 patients with a median follow-up of 21 months, where the 2-year local progression-free survival (L-PFS) was $91.1 \%$ (96.7\% for primary and $83.8 \%$ for recurrent tumors) [31].

However, there is a major concern about this approach, as a short-course scheme of radiotherapy might not be optimal for a palliative intention. A randomized, phase III trial published by Hoskin et al. (FORT trial) has proven the worth of radical doses against short-course radiotherapy. A total of 614 sites were randomized to receive either 24 Gy or 4 Gy at 2 Gy per fraction $(n=299$ and $n=315$, respectively). After a median follow-up of 26 months, 21 and 70 local progressions had developed in each group, respectively. Therefore, the radical dose approach should still be considered as the standard of care for these cases scenario [32].

Based on these findings, this novel approach intended to shorten the treatment course without compromising the effectivity achieved by the standard treatment, by calculating an equivalent radical dose as previously discussed. Koren et al. performed a dosimetric study on electronic brachytherapy with low energy (kilovoltage, kV) $\mathrm{X}$-rays, and showed that irradiation of ocular structures is expected to be safe [33]. In consequence, we initiated a phase I/II study on adjuvant electronic brachytherapy for patients with resected squamous cell carcinoma of the ocular conjunctiva. At single doses between 18 and $22 \mathrm{~Gy}$, we did not see any grade 2 or higher toxicity, which suggested that this therapeutic strategy may also be safe for patients with conjunctival lymphomas, where even lower doses were applied [34].

Finding efficient single-session treatments will also have socio-economic implications in countries such as Peru. The current Peruvian health system aims at optimization of resources while maintaining quality with adequate therapeutic outcomes to guarantee greater access to health services. However, a major challenge is the scarcity of oncologic services in the country, forcing existing facilities to handle a large influx of patients. Obtaining comparable results from treatments that require only a single shot versus multiple applications of EBRT, will be the key to overcome these difficulties. The displacement of patients from their place of origin, assuming transportation and lodging expenses that are not generally contemplated in health coverage, are additional problems to be considered [35]. As for future directions, 
and based on the encouraging outcomes described here, a single-fraction dose de-escalation should be addressed for further evaluation.

Overall, we report a small series of patients with early-stage conjunctival lymphoma treated with a single fraction low-energy electronic brachytherapy. With follow-up periods of more than two years in all cases presented, no evident acute toxicity and promising local control in all patients treated were so far observed. A prospective evaluation in a larger collective is warranted.

\section{Ethics}

The study was approved by the ethics committee at Instituto Nacional de Enfermedades Neoplasicas, Lima, Peru. All patients gave informed consent prior to treatment.

\section{Acknowledgements}

The support of the outpatient clinic staff of the Instituto Nacional de Enfermedades Neoplasicas and the outpatient clinic staff of INEN shall be gratefully acknowledged.

Preliminary data presented at ESTRO 2019, Milan, Italy.

\section{Disclosure}

GRS has received personal fees from Carl Zeiss Meditec outside of the present work. FAG was consultant and speaker for Carl Zeiss Meditec AG, NOXXON Pharma AG, MSD Sharp, and Dohme, Roche Pharma AG, and holds patents related with Carl Zeiss Meditec AG.

The authors report no conflict of interest.

\section{References}

1. Freeman C, Berg JW, Cutler SJ. Occurrence and prognosis of extranodal lymphomas. Cancer 1972; 29: 252-260.

2. Coupland SE, Hellmich M, Auw-Haedrich C et al. Prognostic value of cell-cycle markers in ocular adnexal lymphoma: an assessment of 230 cases. Graefes Arch Clin Exp Ophthalmol 2004; 242: 130-145.

3. Ferry JA, Fung CY, Zukerberg L et al. Lymphoma of the ocular adnexa: A study of 353 cases. Am J Surg Pathol 2007; 31: 170-184.

4. Sjö LD, Ralfkiaer E, Prause JU et al. Increasing incidence of ophthalmic lymphoma in Denmark from 1980 to 2005. Invest Ophthalmol Vis Sci 2008; 49: 3283-3288.

5. Aspiotis M, Gorezis S, Asproudis I et al. Primary mantle cell lymphoma of the conjunctiva: a case report. Virchows Arch 2006; 449: 472-475.

6. Cai J, Liu X, Cheng J et al. MicroRNA-200 is commonly repressed in conjunctival MALT lymphoma, and targets cyclin E2. Graefes Arch Clin Exp Ophthalmol 2012; 250: 523-531.

7. Takada S, Yoshino T, Taniwaki M et al. Involvement of the chromosomal translocation $\mathrm{t}(11 ; 18)$ in some mucosa-associated lymphoid tissue lymphomas and diffuse large B-cell lymphomas of the ocular adnexa: evidence from multiplex reverse transcriptase-polymerase chain reaction and fluorescence in situ hybridization on using formalin-fixed, paraffin-embedded specimens. Mod Pathol 2003; 16: 445-452.

8. Stefanovic A, Lossos IS. Extranodal marginal zone lymphoma of the ocular adnexa. Blood 2009; 114: 501-510.
9. Decaudin D, de Cremoux P, Vincent-Salomon A et al. Ocular adnexal lymphoma: a review of clinicopathologic features and treatment options. Blood 2006; 108: 1451-1460.

10. Liu Q, Schneider F, Ma L et al. Relative Biologic Effectiveness (RBE) of $50 \mathrm{kV}$ X-rays measured in a phantom for intraoperative tumor-bed irradiation. Int J Radiat Oncol Biol Phys 2013; 85: 1127-1133.

11. Wilder RB, Tucker SL, Ha CS et al. Dose-response analysis for radiotherapy delivered to patients with intermediate-grade and large-cell immunoblastic lymphomas that have completely responded to $\mathrm{CHOP}$-based induction chemotherapy. Int J Radiat Oncol Biol Phys 2001; 49: 17-22.

12. Abd Al-Kader L, Sato Y, Takata K et al. A case of conjunctival follicular lymphoma mimicking mucosa-associated lymphoid tissue lymphoma. J Clin Exp Hematop 2013; 53: 49-52.

13. Blasi MA, Tiberti AC, Valente $P$ et al. Intralesional interferon-alpha for conjunctival mucosa-associated lymphoid tissue lymphoma: long-term results. Ophthalmology 2012; 119: 494-500.

14. Matsuo T, Yoshino T. Long-term follow-up results of observation or radiation for conjunctival malignant lymphoma. Ophthalmology 2004; 111: 1233-1237.

15. Cahill M, Barnes C, Moriarty P et al. Ocular adnexal lymphoma - comparison of MALT lymphoma with other histological types. Br J Ophthalmol 1999; 83: 742-747.

16. Holds J, Buchanan A, Hanson R. Intralesional interferon-alpha for the treatment of bilateral conjunctival mucosa-associated lymphoid tissue lymphoma. Pediatr Blood Cancer 2012; 59: 176-178.

17. Lee MJ, Kim N, Choe JY et al. Clinicopathological analysis of ocular adnexal extranodal marginal zone B-cell lymphoma with IgG4-positive cells. PLoS One 2015; 10: e0131458.

18. Nückel H, Meller D, Steuhl KP et al. Anti-CD20 monoclonal antibody therapy in relapsed MALT lymphoma of the conjunctiva. Eur J Haematol 2004; 73: 258-262.

19. Kirkegaard MM, Coupland SE, Prause JU et al. Malignant lymphoma of the conjunctiva. Surv Ophthalmol 2015; 60: 444-458.

20. Bardenstein DS. Ocular adnexal lymphoma: classification, clinical disease, and molecular biology. Ophthalmol Clin North Am 2005; 18: 187-197, x.

21. Meunier J, Lumbroso-Le Rouic L, Vincent-Salomon A et al Ophthalmologic and intraocular non-Hodgkin's lymphoma: a large single centre study of initial characteristics, natural history, and prognostic factors. Hematol Oncol 2004; 22: 143-158.

22. Uno $\mathrm{T}$, Isobe $\mathrm{K}$, Shikama $\mathrm{N}$ et al. Radiotherapy for extranodal, marginal zone, B-cell lymphoma of mucosa-associated lymphoid tissue originating in the ocular adnexa: a multiinstitutional, retrospective review of 50 patients. Cancer 2003; 98: 865-871.

23. Bianciotto C, Shields CL, Lally SE et al. CyberKnife radiosurgery for the treatment of intraocular and periocular lymphoma. Arch Ophthalmol 2010; 128: 1561-1567.

24. Laskar S, Gurram L, Laskar SG et al. Superficial ocular malignancies treated with strontium-90 brachytherapy: long term outcomes. J Contemp Brachytherapy 2015; 7: 369-373.

25. Marr BP, Abramson DH, Cohen GN et al. Intraoperative high-dose rate of radioactive phosphorus 32 brachytherapy for diffuse recalcitrant conjunctival neoplasms: a retrospective case series and report of toxicity. JAMA Ophthalmol 2015; 133: 283-289.

26. Regueiro CA, Valcárcel FJ, Romero J et al. Treatment of conjunctival lymphomas by beta-ray brachytherapy using a strontium-90-yttrium-90 applicator. Clin Oncol 2002; 14: 459-463.

27. Esmaeli B, McLaughlin P, Pro B et al. Prospective trial of targeted radioimmunotherapy with Y-90 ibritumomab tiuxetan 
(Zevalin) for front-line treatment of early-stage extranodal indolent ocular adnexal lymphoma. Ann Oncol 2009; 20: 709714.

28. Fasola CE, Jones JC, Huang DD et al. Low-dose radiation therapy $(2 \mathrm{~Gy} \times 2)$ in the treatment of orbital lymphoma. Int J Radiat Oncol Biol Phys 2013; 86: 930-935.

29. Cotting-Bodmer S, Moulin A, Herbort CP, Jr. et al. Can low dose radiation therapy $(2 \times 2$ gy) be used in primary bilateral conjunctival follicular lymphoma? Klin Monbl Augenheilkd 2016; 233: 524-526.

30. König L, Stade R, Rieber J et al. Radiotherapy of indolent orbital lymphomas: Two radiation concepts. Strahlenther Onkol 2016; 192: 414-421.

31. König L, Hörner-Rieber J, Bernhardt D et al. Response rates and recurrence patterns after low-dose radiotherapy with 4 Gy in patients with low-grade lymphomas. Strahlenther Onkol 2018; 194: 454-461.

32. Hoskin PJ, Kirkwood AA, Popova B et al. 4 Gy versus 24 Gy radiotherapy for patients with indolent lymphoma (FORT): a randomised phase 3 non-inferiority trial. Lancet Oncol 2014; 15: 457-463.

33. Koren S, Bragilovski D, Tafo AG et al. SU-E-T-55: A novel applicator for high dose rate brachytherapy treatments of ocular conjunctiva. Med Phys 2015; 42 (6 Part 12): 3343.

34. Sarria GR, Sarria GJ, Rivera PF et al. Phase I/II study on kilovoltage surface brachytherapy in conjunctival cancer: preliminary results. Ecancermedicalscience 2018; 12: 835.

35. Sarria GJ, Saria GR, Pinillos LV. The Inca Trail to the present: the development of radiation therapy in Peru. Int J Radiat Oncol Biol Phys 2018; 101: 244-249. 